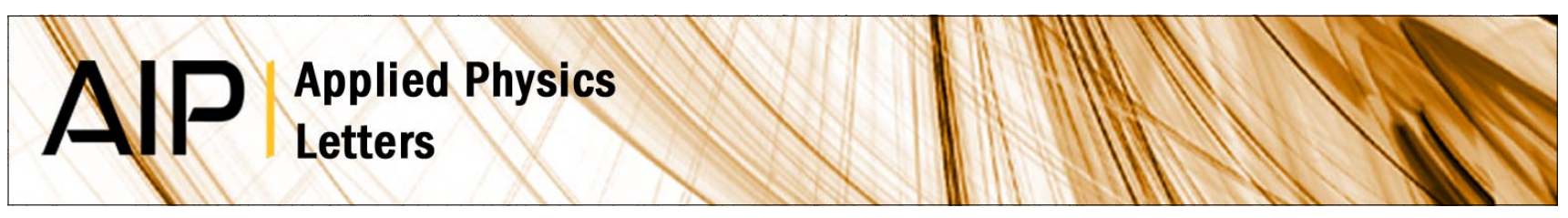

\title{
Electron spin filtering by thin GaNAs/GaAs multiquantum wells
}

Y. Puttisong, X. J. Wang, I. A. Buyanova, H. Carrére, F. Zhao et al.

Citation: Appl. Phys. Lett. 96, 052104 (2010); doi: 10.1063/1.3299015

View online: http://dx.doi.org/10.1063/1.3299015

View Table of Contents: http://apl.aip.org/resource/1/APPLAB/v96/i5

Published by the American Institute of Physics.

Additional information on Appl. Phys. Lett.

Journal Homepage: http://apl.aip.org/

Journal Information: http://apl.aip.org/about/about_the_journal

Top downloads: http://apl.aip.org/features/most_downloaded

Information for Authors: http://apl.aip.org/authors

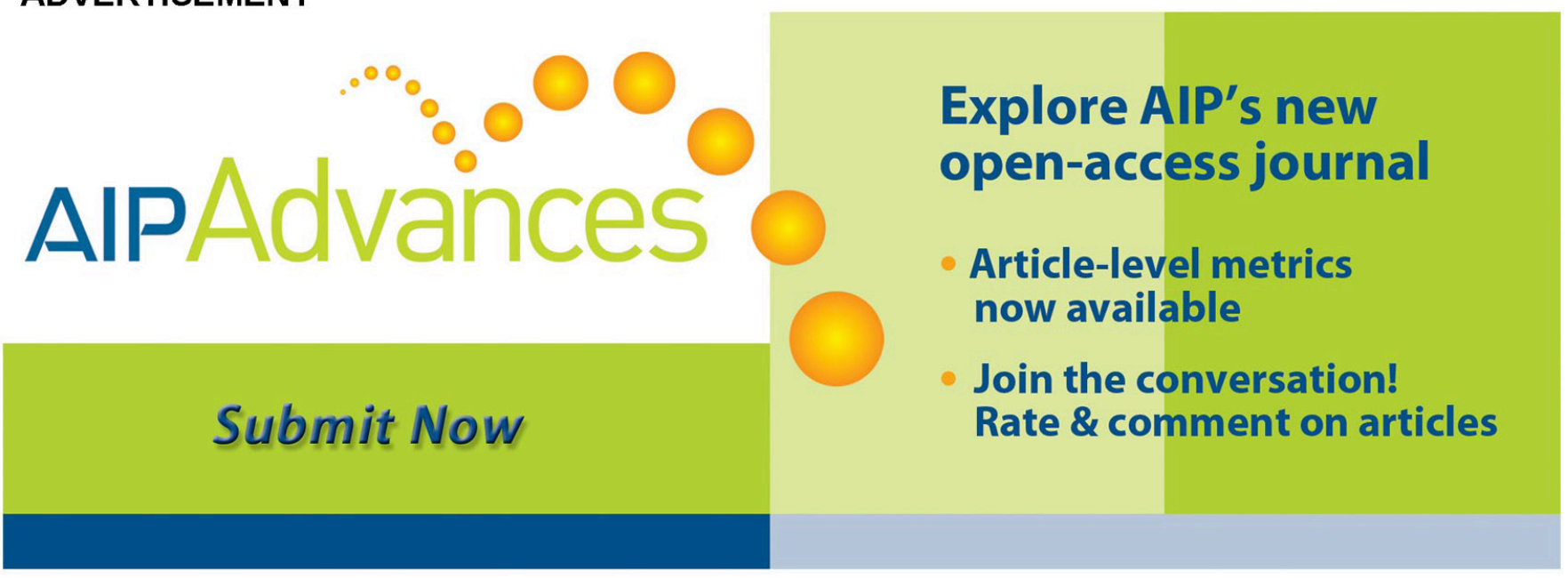




\title{
Electron spin filtering by thin GaNAs/GaAs multiquantum wells
}

\author{
Y. Puttisong, ${ }^{1}$ X. J. Wang, ${ }^{1}$ I. A. Buyanova, ${ }^{1}$ H. Carrére, ${ }^{2}$ F. Zhao, ${ }^{2}$ A. Balocchi, ${ }^{2}$ \\ X. Marie, ${ }^{2}$ C. W. Tu, ${ }^{3}$ and W. M. Chen ${ }^{1, a)}$ \\ ${ }^{1}$ Department of Physics, Chemistry, and Biology, Linköping University, S-581 83 Linköping, Sweden \\ ${ }^{2}$ Université de Toulouse, LPCNO: INSA, UPS, CNRS, 135 avenue de Rangueil, \\ 31077 Toulouse Cedex, France \\ ${ }^{3}$ Department of Electrical and Computer Engineering, University of California, La Jolla, \\ California 92093, USA
}

(Received 30 November 2009; accepted 6 January 2010; published online 1 February 2010)

\begin{abstract}
Effectiveness of the recently discovered defect-engineered spin-filtering effect is closely examined in GaNAs/GaAs multiquantum wells (QWs) as a function of QW width. In spite of narrow well widths of 3-9 nm, rather efficient spin filtering is achieved at room temperature. It leads to electron spin polarization larger than $18 \%$ and an increase in photoluminescence intensity by $65 \%$ in the $9 \mathrm{~nm}$ wide QWs. A weaker spin filtering effect is observed in the narrower QWs, mainly due to a reduced sheet concentration of spin-filtering defects (e.g., $\mathrm{Ga}_{\mathrm{i}}$ interstitial defects). () 2010 American Institute of Physics. [doi:10.1063/1.3299015]
\end{abstract}

Spin, an extra degree of freedom to electron charge, has led to exciting ideas of spintronic devices and spin-based quantum information processors and memories. ${ }^{1-4}$ Essential to these devices is our ability to generate electron spin polarization by a spin aligner or a spin filter. During the past decade, we have witnessed a rapid progress in developments of spin filters based on, e.g., diluted magnetic semiconductors. ${ }^{5,6}$ Recent calculations suggested spin filters based on nonmagnetic semiconductors ${ }^{7,8}$ as an attractive alternative because of their immediate compatibility with existing semiconductor technology and the absence of harmful side effects accompanying introduction of magnetic impurities. Most recently, Wang et al. ${ }^{9}$ reported that spin-dependent recombination (SDR) processes via spin-filtering $\mathrm{Ga}_{\mathrm{i}}$ selfinterstitial defects can transform nonmagnetic $\mathrm{Ga}(\mathrm{In}) \mathrm{NAs}$ into an efficient spin filter operating at room temperature (RT) without applying a magnetic field, i.e., under conditions desirable for device applications. Spin polarization of conduction band (CB) electrons as high as $32 \%$ can be achieved in thick GaNAs epilayers. ${ }^{9}$ The actual value could be even higher if one takes into account the fact that optical polarization $\mathrm{P}_{\mathrm{o}}$ of the band-to-band (BB) transition, which was employed to monitor electron spin polarization $\mathrm{P}_{\mathrm{e}}$, could severely underestimate $\mathrm{P}_{e}$ as a result of strong mixing of heavy-hole (hh) and light-hole (lh) valence band (VB) states and also a spectral overlap between the e-hh and e-lh BB transitions.

It is well known that modern semiconductor electronic and optoelectronic devices are nearly exclusively based on thin layered and quantum structures grown by epitaxial techniques. To add the spin degree of freedom to these devices for future multifunctional applications, spin-enabled functionality must be effective in quantum and nano structures. The aim of this work is to address this issue by investigating spin-filtering properties in GaNAs/GaAs quantum wells (QWs) with a width $\left(\mathrm{L}_{\mathrm{z}}\right)$ in the range of 3-9 $\mathrm{nm}$.

The studied QWs were grown by molecular beam epitaxy on a (001)-oriented semi-insulating or $\mathrm{n}^{+}$GaAs sub-

${ }^{a)}$ Electronic mail: wmc@ifm.liu.se. strate, and were capped by a $250 \mathrm{~nm}$ thick GaAs layer. All structures contain seven periods of GaNAs/GaAs QWs with $[\mathrm{N}]=1.6 \%$ and $\mathrm{L}_{\mathrm{z}}=3,5,7$, and $9 \mathrm{~nm}$, sandwiched between $20.2 \mathrm{~nm}$ GaAs barriers. Both continuous-wave (cw) and time-resolved optical orientation techniques were employed at RT. A laser beam propagating parallel to the growth axis, from a cw Ti-sapphire laser or a mode-locked Ti:sapphire laser (with a pulse width of $1.5 \mathrm{ps}$ and a repetition frequency of $80 \mathrm{MHz}$ ), was used as an excitation source. Typical excitation power was up to $300 \mathrm{~mW}$ (cw laser) and $100 \mathrm{~mW}$ (pulsed laser), focused on a spot of approximately $0.1-1 \mathrm{~mm}$. The excitation wavelengths of $832 \mathrm{~nm}(\mathrm{cw})$ and $790 \mathrm{~nm}$ (pulsed) were chosen to induce BB absorption involving hh and $\mathrm{lh}$ in GaAs, such that a preferential spin orientation of CB electrons can be created with a maximum value of $\left|\mathrm{P}_{\mathrm{e}}\right|$ $=50 \% .{ }^{10}$ Under such excitation conditions, most of the carriers participating in the $\mathrm{BB}$ recombination in the GaNAs QWs were injected from the GaAs barriers and the cap layer. Resulting photoluminescence (PL) was detected in a backscattering geometry by a charge-coupled device in cw experiments or a streak camera with an overall time-resolution of $8 \mathrm{ps}$ in time-resolved experiments. Circular-polarization of excitation light was provided and that of PL was analyzed by using a quarter-wave plate together with a linear polarizer. Optically detected magnetic resonance (ODMR) experiments were done at $9.14 \mathrm{GHz}$ and $4 \mathrm{~K}$, by detecting spin-flip induced changes of the BB PL emission.

The principle of an SDR process via a spin-filtering defect such as a $\mathrm{Ga}_{\mathrm{i}}$ defect is schematically illustrated in Figs. 1(a) and 1(b). ${ }^{9}$ Under linear excitation $\left(\sigma^{\mathrm{x}}\right)$, no preferred spin orientation is created for $\mathrm{CB}$ electrons or the electron bound to the paramagnetic defect. As a result, the defect randomly and equally depletes photogenerated $\mathrm{CB}$ electrons of both spin orientations, resulting in fewer carriers participating in the $\mathrm{BB}$ transition. In contrast, under circular excitation $\left(\sigma^{+}\right.$or $\sigma^{-}$), CB electrons are photogenerated with a preferential spin orientation. ${ }^{10}$ This subsequently drives the defect electron to the same preferred spin orientation via dynamic spin polarization, ${ }^{9,11-14}$ yielding spin blockade of further capture of $\mathrm{CB}$ electrons and thus an enhanced $\mathrm{BB}$ transition. Once a $\mathrm{CB}$ electron undergoes a spin flip, it will rapidly be depleted 
(a)

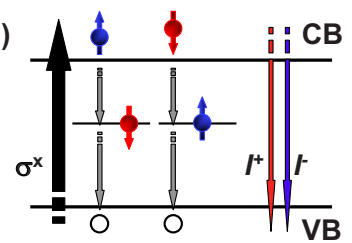

(b)

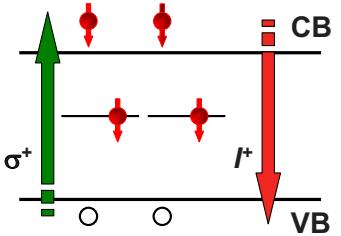

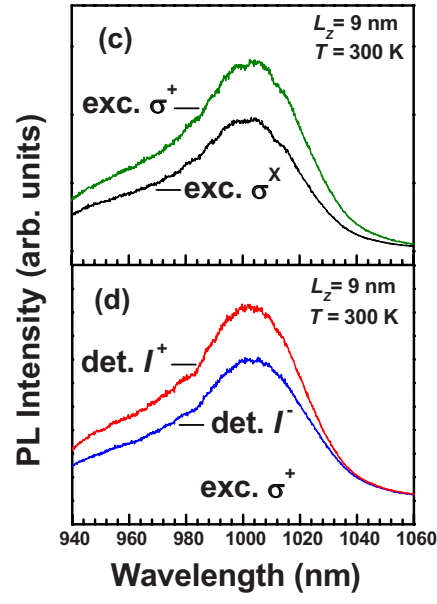

FIG. 1. (Color online) Carrier capture and recombination processes via spinfiltering defects and BB transition under linearly (a) or circularly (b) polarized optical excitation, when the defect-engineered spin-filtering effect is inactive and active, respectively. (c) Typical PL spectra of the studied GaNAs/GaAs QWs with $[\mathrm{N}]=1.6 \%$, obtained by detecting total PL intensity (i.e., $\mathrm{I}^{+}+\mathrm{I}^{-}$) at RT under linearly and circularly polarized excitation. (d) PL spectra at RT under $\sigma^{+}$excitation by detecting $\mathrm{I}^{+}$or $\mathrm{I}^{-}$.

by the defect such that a high degree of spin polarization of $\mathrm{CB}$ electrons is maintained. ${ }^{13}$ Such spin-filtering effect is manifested by a higher intensity and polarization of the $\mathrm{BB}$ PL.

Representative BB PL spectra from the studied QWs are displayed in Figs. 1(c) and 1(d). The spin-filtering effect is distinctly evident from (i) stronger BB PL intensity under $\sigma^{+}$ excitation than under $\sigma^{\mathrm{x}}$ excitation [Fig. 1(c)] and (ii) stronger $\sigma^{+}$-polarized $\mathrm{PL}$ component $\left(\mathrm{I}^{+}\right)$as compared with $\sigma^{-}$-polarized component $\left(\mathrm{I}^{-}\right)$under $\sigma^{+}$excitation [Fig. 1(d)]. A summary of the results from the QWs with different widths is given in Fig. 2(a). To quantify the spin-blockade effect, the results are presented in terms of an SDR ratio, defined as $\mathrm{I}\left(\sigma^{+}\right.$or $\left.\sigma^{-}\right) / \mathrm{I}\left(\sigma^{\mathrm{x}}\right)$ where $\mathrm{I}\left(\sigma^{+}\right.$or $\left.\sigma^{-}\right)$and $\mathrm{I}\left(\sigma^{\mathrm{x}}\right)$ are total BB PL intensities under circular and linear excitation, respectively. The spin-filtering effect and the resulting $\mathrm{CB}$ electron spin polarization is assessed from PL polarization
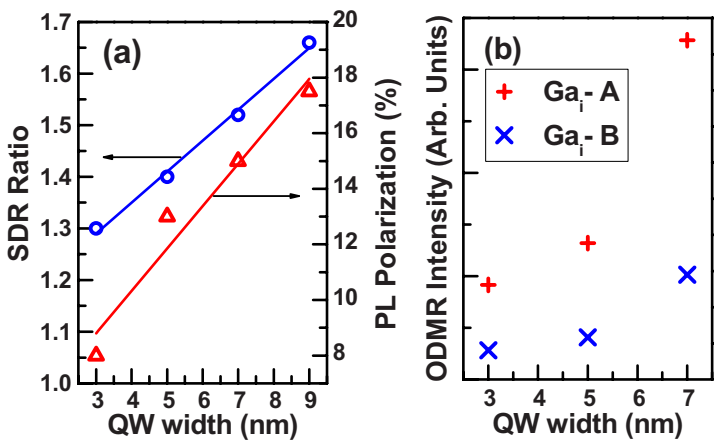

FIG. 2. (Color online) (a) Values of SDR ratio and PL polarization as a function of QW width, obtained at RT under the same excitation power. They represent the maximum values measured over the spectral range of the BB PL emission. The symbols are experimental data and the lines are guides to the eye. (b) ODMR intensities of the spin-filtering $\mathrm{Ga}_{\mathrm{i}}$ interstitial defects as a function of $\mathrm{QW}$ width. The $\mathrm{Ga}_{\mathrm{i}}$ interstitial defect denoted by $\mathrm{Ga}_{\mathrm{i}}-\mathrm{A}$ $\left(\mathrm{Ga}_{\mathrm{i}} \mathrm{-B}\right)$ is characterized by its spin Hamiltonian parameters $\mathrm{g}=2.005$ (2.000), $\quad \mathrm{A}\left({ }^{69} \mathrm{Ga}\right)=750 \times 10^{-4} \mathrm{~cm}^{-1}\left(1230 \times 10^{-4} \mathrm{~cm}^{-1}\right)$ and $\mathrm{A}\left({ }^{71} \mathrm{Ga}\right)$ $=952.5 \times 10^{-4} \mathrm{~cm}^{-1}\left(1562 \times 10^{-4} \mathrm{~cm}^{-1}\right)$, obtained from a best fit of the spin Hamiltonian $H=\boldsymbol{\mu}_{\mathrm{B}} \mathrm{g} \mathbf{B} \cdot \mathbf{S}+\mathrm{A} \boldsymbol{S} \cdot \mathbf{I}$ to the experimental ODMR data as described in Ref. 9.
$\mathrm{P}_{\mathrm{o}}=\left(\mathrm{I}^{+}-\mathrm{I}^{-}\right) /\left(\mathrm{I}^{+}+\mathrm{I}^{-}\right)$. A monotonous increase in the SDR ratio from 1.3 to 1.66 was clearly observed with increasing $\mathrm{L}_{\mathrm{z}}$ from 3 to $9 \mathrm{~nm}$. This spin-blockade effect is closely correlated with the spin-filtering effect, evident from an increase in $\mathrm{P}_{\mathrm{o}}$ from $8 \%$ to $18 \%$. It should be pointed out that $\mathrm{P}_{\mathrm{o}}=-\mathrm{P}_{\mathrm{e}}\left(\right.$ or $\mathrm{P}_{\mathrm{o}}=+\mathrm{P}_{\mathrm{e}}$ ) when only the e-hh (or e-lh) emission is monitored and there is no hh-lh mixing. The measured $\mathrm{P}_{\mathrm{o}}$ values were obtained by monitoring the shorter wavelength side of the BB PL emission, which is dominated by the e-hh emission, to minimize the spectral overlap with the longerwavelength e-lh emission and resulting compensation in optical polarization. However, at such higher detection energies, hh-lh mixing becomes stronger and could in principle lead to an underestimate of $\mathrm{P}_{\mathrm{e}}$ by up to $50 \% .^{15}$ The measured $\mathrm{P}_{\mathrm{o}}$ values thus represent the low bound for $\mathrm{P}_{\mathrm{e}}$. Nevertheless, the trend of increasing $\mathrm{P}_{e}$ with increasing $\mathrm{L}_{z}$ is apparent. Different from $P_{e}$, the SDR ratio does not suffer from such complications because it monitors the total BB PL intensity (i.e., $\mathrm{I}^{+}+\mathrm{I}^{-}$) and no compensation in optical polarization occurs in this case.

The observed increases in the SDR ratio and $\mathrm{P}_{\mathrm{e}}$ with increasing $\mathrm{L}_{\mathrm{z}}$ reveal stronger effects of spin blockade and spin filtering in the wider QWs. In principle, several factors can contribute to this trend. First, the radiative recombination of the $\mathrm{BB}$ transition can accelerate with decreasing $\mathrm{L}_{\mathrm{z}}$ due to a stronger overlap of wave functions between $\mathrm{CB}$ electrons and VB holes. It competes with the SDR via the spinfiltering defects and could undermine the latter. Second, spin relaxation of $\mathrm{CB}$ electrons may vary with $\mathrm{L}_{\mathrm{z}}$ under the D'yakonov-Perel (DP) mechanism. ${ }^{16-18}$ If spin relaxation rate becomes comparable with or exceeds the capture rate of $\mathrm{CB}$ electrons by the defects, the latter will no longer be able to catch up with spin relaxation and to deplete spin-flipped $\mathrm{CB}$ electrons, leading to a reduction in spin-filtering efficiency. Third, if the unit-volume density of the defects remains the same in all QWs, which is determined by the identical growth conditions and $\mathrm{N}$ composition, the area (or sheet) concentration (and thus the total number) of the spinfiltering defects increases with increasing $\mathrm{L}_{\mathrm{z}}$. Thus, twodimensional (2D) CB electrons in the wider QWs are more probable to be spin-filtered by the defects than that in the narrower QWs.

To examine relative importance of the aforementioned mechanisms, we employed time-resolved optical orientation at RT. The results from the QWs with $\mathrm{L}_{\mathrm{z}}=3$ and $9 \mathrm{~nm}$ under linear and circular excitation are shown in Figs. 3(a) and 3(b). If the BB PL were governed by the radiative recombination, a faster decay would be expected for the narrower QWs in the absence of spin blockade under linear excitation. This contradicts with our experimental observation of an overall faster decay in the $9 \mathrm{~nm}$ QWs than that in the $3 \mathrm{~nm}$ QWs. This finding is consistent with the assumption that carrier recombination is dominated by the SDR process via the defects, if spin-filtering should be effective. ${ }^{9}$ The radiative recombination of the $\mathrm{BB}$ transition must be much less efficient as compared with the SDR process, and therefore its modification by quantum confinement cannot be the reason for the observed change in spin filtering between the QWs with different $\mathrm{L}_{\mathrm{z}}$.

The observed transient behavior of the BB PL under circular excitation can be characterized by two decay components, ${ }^{19}$ distinctive to the physical processes involved 


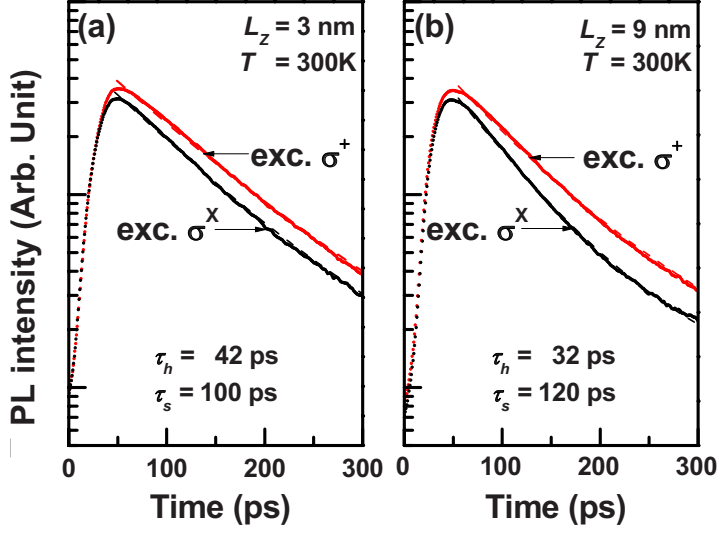

FIG. 3. (Color online) PL decays of the GaNAs/GaAs QWs with $\mathrm{L}_{z}=3$ (a) and $9 \mathrm{~nm}$ (b), obtained at RT under circularly and linearly polarized excitation. The dashed lines are the fitting curves with the specified time constants.

in the SDR. Immediately after the laser pulses, photoexcited electrons were quickly captured by the paramagnetic defects, which is too fast to be resolved within our instrument limit of $8 \mathrm{ps}$. The subsequent capture of holes by the defects, after each of the defects has been occupied by two electrons, gives rise to the observable fast PL decay component $\left[\propto \exp \left(-2 \mathrm{t} / \tau_{\mathrm{h}}\right)(\right.$ Ref. 19)] shown in Fig. 3. During the processes, dynamic spin polarization takes place for the defect electrons leading to spin blockade of further carrier capture and recombination via the defects. After that, carriers can only be captured by the defects upon spin flips of CB electrons, characterized by a spin relaxation time $\tau_{\mathrm{s}}$, which corresponds to the slow PL decay component $\left[\propto \exp \left(-2 \mathrm{t} / \tau_{\mathrm{s}}\right)\right.$ (Ref. 19)] in Fig. 3. Whereas the fast PL decay process occurs under both circular and linear excitation, the spin relaxation process is only important under the condition of spin blockade, explaining the difference between the PL decays under circular and linear excitation. From a best fit of the experimental data, $\tau_{\mathrm{s}}=100 \pm 10 \mathrm{ps}$ and $120 \pm 10 \mathrm{ps}$ were obtained for the QWs with $\mathrm{L}_{\mathrm{z}}=3$ and $9 \mathrm{~nm}$, respectively. This variation in $\tau_{\mathrm{s}}$ with $\mathrm{L}_{\mathrm{z}}$, expected from the DP spin relaxation mechanism, is in agreement with earlier results from GaAs QWs. ${ }^{18}$ In any case, a change from $\tau_{\mathrm{s}}=120$ to $100 \mathrm{ps}$ can only decrease the SDR ratio from 1.66 to 1.5 estimated from a rate equation analysis. ${ }^{9,14}$ This cannot account for a reduction from 1.66 to 1.3 observed in our experiments [Fig. 2(a)], excluding a change in spin relaxation of $\mathrm{CB}$ electrons as the dominant mechanism for the observed change in the spin-filtering efficiency between the QWs.

As the $\mathrm{BB}$ radiative recombination and $\mathrm{CB}$ electron spin relaxation are both insufficient to explain our experimental findings, a change in the sheet concentration of the spinfiltering defects could be the most likely cause. This is in fact indicated by the fitting parameters of $\tau_{\mathrm{h}}=42 \pm 4 \mathrm{ps}$ and $32 \pm 4$ ps for the QWs with $\mathrm{L}_{\mathrm{z}}=3$ and $9 \mathrm{~nm}$, respectively. As $\tau_{\mathrm{h}}=1 / \gamma_{\mathrm{h}} N_{\uparrow \downarrow}{ }_{\uparrow},{ }^{9,14}$ where $\gamma_{\mathrm{h}}$ is a hole capture coefficient and $N_{\uparrow \downarrow}$ the concentration of the defects occupied by two electrons, the shorter $\tau_{\mathrm{h}}$ in the wider QWs evidences for a higher defect concentration that can lead to stronger spin-filtering effect. A rate equation analysis supports that the measured difference in $\tau_{\mathrm{h}}$ is sufficient to account for the difference in the SDR ratio between the two QWs. This conclusion was further confirmed by our ODMR results, which show that ODMR intensities of the spin-filtering defects ${ }^{9}$ (i.e., $\mathrm{Ga}_{\mathrm{i}}$ interstitial defects denoted by $\mathrm{Ga}_{\mathrm{i}}-\mathrm{A}$ and $\mathrm{Ga}_{\mathrm{i}} \mathrm{B}$ ) increase with increasing $\mathrm{L}_{z}$ [Fig. 2(b)]. As the ODMR intensity scales with the $2 \mathrm{D}$ carrier capture and recombination rate via the defects, ${ }^{9,20}$ which is proportional to the sheet defect concentrations, the observed increase of the ODMR intensities signifies a corresponding increase in the sheet concentrations (and thus the total numbers) of the spin-filtering defects with increasing $\mathrm{L}_{\mathrm{z}}$.

In conclusion, the defect-engineered spin-filtering effect has been shown to be effective even in GaNAs QWs as narrow as $3 \mathrm{~nm}$. The effect becomes stronger in the wider QWs and is shown to be mainly due to an increase in the sheet concentrations of the spin-filtering defects. Effects of quantum confinement on spin-filtering caused by changes of rates in $\mathrm{BB}$ carrier recombination and in $\mathrm{CB}$ electron spin relaxation are shown to play a less important role. The results provide a useful guideline for improving spin-filtering efficiency in quantum and nanostructures by means of increasing the concentration of the spin-filtering defects.

${ }^{1}$ G. Prinz, Science 282, 1660 (1998).

${ }^{2}$ S. A. Wolf, D. D. Awschalom, R. A. Buhrman, J. M. Daughton, S. von Molnár, M. L. Roukes, A. Y. Chtchelkanova, and D. M. Treger, Science 294, 1488 (2001)

${ }^{3}$ I. Žutić, J. Fabian, and S. Das Sarma, Rev. Mod. Phys. 76, 323 (2004).

${ }^{4}$ C. Chappert, A. Fert, and F. Nguyen Van Dau, Nature Mater. 6, 813 (2007).

${ }^{5}$ R. Fiederling, M. Kleim, G. Reuscher, W. Ossau, G. Schmidt, A. Waag, and L. W. Molenkamp, Nature (London) 402, 787 (1999).

${ }^{6}$ Y. Ohno, D. K. Young, B. Beschoten, F. Matsukura, H. Ohno, and D. D. Awschalom, Nature (London) 402, 790 (1999).

${ }^{7}$ T. Koga, J. Nitta, and H. Takayanagi, Phys. Rev. Lett. 88, 126601 (2002).

${ }^{8}$ D. Z.-Y. Ting and X. Cartoixá, Appl. Phys. Lett. 81, 4198 (2002).

${ }^{9}$ X. J. Wang, I. A. Buyanova, F. Zhao, D. Lagarde, A. Balocchi, X. Marie, C. W. Tu, J. C. Harmand, and W. M. Chen, Nature Mater. 8, 198 (2009).

${ }^{10}$ F. Meier and B. P. Zakharchenya, Optical Orientation (North-Holland, Amsterdam, 1984).

${ }^{11}$ C. Weisbuch and G. Lampel, Solid State Commun. 14, 141 (1974).

${ }^{12}$ D. Paget, Phys. Rev. B 30, 931 (1984).

${ }^{13}$ V. K. Kalevich, E. L. Ivchenko, M. M. Afanasiev, A. Yu. Shiryaev, A. Yu. Egorov, V. M. Ustinov, B. Pal, and Y. Masumoto, JETP Lett. 82, 455 (2005).

${ }^{14}$ D. Lagarde, L. Lombez, X. Marie, A. Balocchi, T. Amand, V. K. Kalevich, A. Shiryaev, E. Ivchenko, and A. Egorov, Phys. Status Solidi A 204, 208 (2007).

${ }^{15}$ V. K. Kalevich, E. L. Ivchenko, A. Yu. Shiryaev, M. M. Afanasiev, A. Yu. Egorov, M. Ikezawa, and Y. Masumoto, Semicond. Sci. Technol. 23, 114008 (2008).

${ }^{16}$ M. I. D'yakonov and V. I. Perel, Fiz. Tverd. Tela 13, 3581 (1971) Sov. Phys. Solid State 13, 3023 (1972).

${ }^{17}$ M. I. D'yakonov and V. Y. Kachorovskii, Sov. Phys. Semicond. 20, 110 (1986).

${ }^{18}$ A. Malinowski, R. S. Britton, T. Grevatt, R. T. Harley, D. A. Ritchie, and M. Y. Simmons, Phys. Rev. B 62, 13034 (2000).

${ }^{19}$ V. K. Kalevich, A. Yu. Shiryaev, E. L. Ivchenko, A. Yu. Egorov, L. Lombez, D. Lagarde, X. Marie, and T. Amand, JETP Lett. 85, 174 (2007).

${ }^{20}$ W. M. Chen, Thin Solid Films 364, 45 (2000). 